\title{
Perspective
}

PERSPECTIVE Actualité en histoire de l'art

1 | 2012

Art et pouvoir

\section{Le portrait du roi : entre art, histoire, anthropologie et sémiologie}

Royal portraiture: between art, history, anthropology, and semiology

Antonio Pinelli, Gérard Sabatier, Barbara Stollberg-Rilinger, Christine Tauber et Diane Bodart

Traducteur : Éric Leroy du Cardonnoy, Pierre Rusch et Renaud Temperini

URL : http://journals.openedition.org/perspective/423

DOI : $10.4000 /$ perspective.423

ISSN : 2269-772

Éditeur

Institut national d'histoire de l'art

Édition imprimée

Date de publication : 30 juin 2012

Pagination : 11-28

ISSN : 1777-7852

Référence électronique

Antonio Pinelli, Gérard Sabatier, Barbara Stollberg-Rilinger, Christine Tauber et Diane Bodart, « Le portrait du roi : entre art, histoire, anthropologie et sémiologie », Perspective [En ligne], 1 | 2012, mis en ligne le 30 décembre 2013, consulté le 01 octobre 2020. URL : http://journals.openedition.org/ perspective/423; DOI : https://doi.org/10.4000/perspective.423 


\section{Débat}

\section{Travaux}

Actualité

\section{Le portrait du roi : entre art, histoire, anthropologie et sémiologie}

Points de vue de Antonio Pinelli, Gérard Sabatier, Barbara Stollberg-Rilinger et Christine Tauber, avec Diane Bodart

Dans la théorie des arts à l'époque moderne, le portrait du roi a un statut singulier : en tant que portrait, il dépend du dictat de la ressemblance au modèle naturel, qui l'éloigne de la noble quête de l'idéal, mais en tant que représentation du souverain, donc d'un personnage hors norme à la perfection incomparable, il a vocation d'être un chef-d'œuvre. De ce fait, le portrait du roi échappe, du moins dans la littérature encomiastique, au discrédit qui frappe progressivement le genre du portrait et aboutit à son déclassement dans la hiérarchie artistique établie au cours du XVII siècle. De surcroît, il participe à sa manière à la revendication de noblesse de la peinture et de la sculpture grâce à la relation directe que sa réalisation instaure entre le portraitiste et le souverain, ce qui en fait un vecteur potentiel de reconnaissance sociale et même d'anoblissement pour les artistes.

Fruit d'une construction visant à concilier le témoignage d'une physionomie naturelle et l'expression d'une dignité suprême, le portrait du roi est un miroir du prince, dans l'acception tant optique que symbolique du terme : il donne à voir, par le truchement d'une image spéculaire du monarque, l'idéal exemplaire des vertus souveraines que celui-ci se doit d'incarner. Expression de la majesté souveraine, le portrait en est aussi l'instrument, car il participe à la représentation du pouvoir au sens large, en contribuant à déterminer l'exercice de l'autorité en acte au même titre que les insignes royaux, tout en lui donnant un visage naturel. Dans le cérémonial politique in absentia, il s'impose comme un substitut du monarque, devant lequel les actes officiels se déroulent et le public se comporte " comme si »le souverain était présent. La valeur de cette présence - juridique, symbolique, "réelle » - varie en fonction des lieux et des circonstances de la monstration du portrait, non sans interférences avec la dimension du sacré, notamment du fait de l'origine iconique de
Diane Bodart, maître de conférences en histoire de l'art à l'université de Poitiers, est l'auteur d'études sur les relations entre art et politique à la période moderne.

Antonio Pinelli, professeur

à l'université de Florence et directeur de la revue Ricerche di Storia dell'arte, est spécialiste de l'art italien du XIV̌e au XVIII' siècle.

Gérard Sabatier, dont les travaux portent notamment sur la monarchie française, est professeur émérite d'histoire moderne à I'université Pierre Mendès France Grenoble II.

Barbara Stollberg-Rilinger enseigne l'histoire moderne à l'université de Münster. Elle étudie les cérémonies et symboles

de l'Ancien Régime, et ses procédures politiques.

Christine Tauber, privatdocent à l'université de Munich et rédactrice en chef de la revue Kunstchronik, travaille notamment sur les rapports art-politique en France à l'époque moderne. 
l'image royale. Dans ce cadre, la force du portrait repose moins sur sa qualité artistique que sur son dispositif de présentation en lieu et place $d u$ monarque : dans le dialogue établi entre le roi représenté par son image en majesté et les sujets reconnaissant l'image comme la représentation de leur roi se noue et se renoue l'union de la couronne. Du renversement de ces mêmes codes sera déterminé en revanche son démembrement, lorsque les gestes d'infamie remplaceront les signes d'honneur qui avaient jusqu'alors confirmé la légitimité de l'autorité souveraine.

Le portrait du roi comme chef-d'œuvre est ainsi investi des pouvoirs politiques propres à toute image royale : il est par excellence un objet d'étude à la croisée des disciplines des sciences humaines - notamment I'histoire de l'art, I'histoire, la sémiologie, I'anthropologie - permettant d'envisager l'œuvre d'art au sein d'une conception plus vaste de l'image. Si la nécessité d'une approche holistique est désormais reconnue, les modalités de son application ne sont pas sans soulever des interrogations, notamment parce que l'image, par rapport au document textuel, exige des outils herméneutiques appropriés à la spécificité de son langage figuratif et à son immanence visuelle. Quant aux pouvoirs de présence qui lui sont attribués, suivant des constantes qui traversent les siècles et les cultures, dans quelle mesure la compréhension des termes de leur efficace et du système sémantique qu'ils induisent, ne relève-t-elle pas en dernier ressort de la spécificité de contextes culturels et historiques ? [Diane Bodart]

Diane Bodart. Le portrait du roi comme cuvre d'art, mais aussi comme instrument d'une relation privilégiée entre le prince et son artiste, a une longue histoire dont le mythe fondateur remonte à Alexandre le Grand et Apelle. Toutefois, bien des aspects du portrait du roi échappent à la dimension de l'art et en font un objet d'étude à la croisée des différentes disciplines, dont l'histoire, l'anthropologie et la sémiologie. Quels sont à vos yeux les principaux apports, mais peut-être aussi les limites, de ce dialogue entre diverses approches $d u$ portrait du roi ?

Christine Tauber. II me semble impossible d'envisager l'analyse du portrait autrement que par le biais transdisciplinaire et grâce à une histoire culturelle et politique de l'art qui réunit, selon une approche holistique, des méthodes artistiques, sociohistoriques, anthropologiques et ethnologiques. Dans les pays germanophones, cette méthode a été développée davantage en histoire qu'en histoire de I'art ${ }^{1}$. Pour saisir le portrait comme genre à mi-chemin entre le politique et l'art, il faut faire appel à l'iconographie politique - approche développée surtout à l'institut Warburg à Hambourg par Martin Warnke et ses élèves ${ }^{2}$ - ainsi qu'à l'histoire des représentations, des rituels et du pouvoir royal. D'un point de vue purement artistique et esthétique, le portrait du monarque est peu instructif, puisque le postulat de la reconnaissance impose des limites très étroites à l'innovation artistique. Ce type de portrait oscille constamment entre deux pôles. D'une part, il est inéluctablement soumis à la mimèsis, ne pouvant remplir sa fonction de portrait si l'individu représenté n'est pas reconnaissable, ce qui peut être causé par le besoin exagéré d'idéalisation et d'hérö̈sation de la personne représentée. D'autre part, une fidélité référentielle trop grande contient le danger d'une perte d'autorité. Le choix de l'artiste de cour chargé de choyer l'image du monarque 
est donc une décision politique de première importance qui ne se saisit que lorsque l'on considère toutes les sphères culturelles déterminantes. Le combat pour la reconnaissance et la tentative de surenchère marquent la représentation des monarques européens à partir la fin du XVe siècle. Dans toutes les cours d'Europe, le portrait était I'un des médias les plus puissants de la politique artistique, faisant la preuve de la force physique et de la supériorité morale du souverain. Les portraits composites maniéristes de Giuseppe Arcimboldo constituent l'apogée de ces stratégies de surenchère mises en place par les cours. L'image de l'empereur Rodolphe II représentée sous les traits de Vertumne, par exemple, est constituée de l'assemblage d'éléments naturels symbolisant I'accumulation de vertus (1591, Stockholm, Skoklosters Slott ; fig. 1) ; I'équilibre entre la mimèsis et I'idéalisation symbolique semble dans ce cas réussi. $C^{\prime}$ est un exemple parmi d'autres qui montre également qu'une histoire et une critique des styles dans le domaine de l'art n'ont pas de sens sans les disciplines voisines que sont I'histoire politique et I'histoire du rituel ${ }^{3}$.

Gérard Sabatier. Face au portrait, I'historien peut avoir

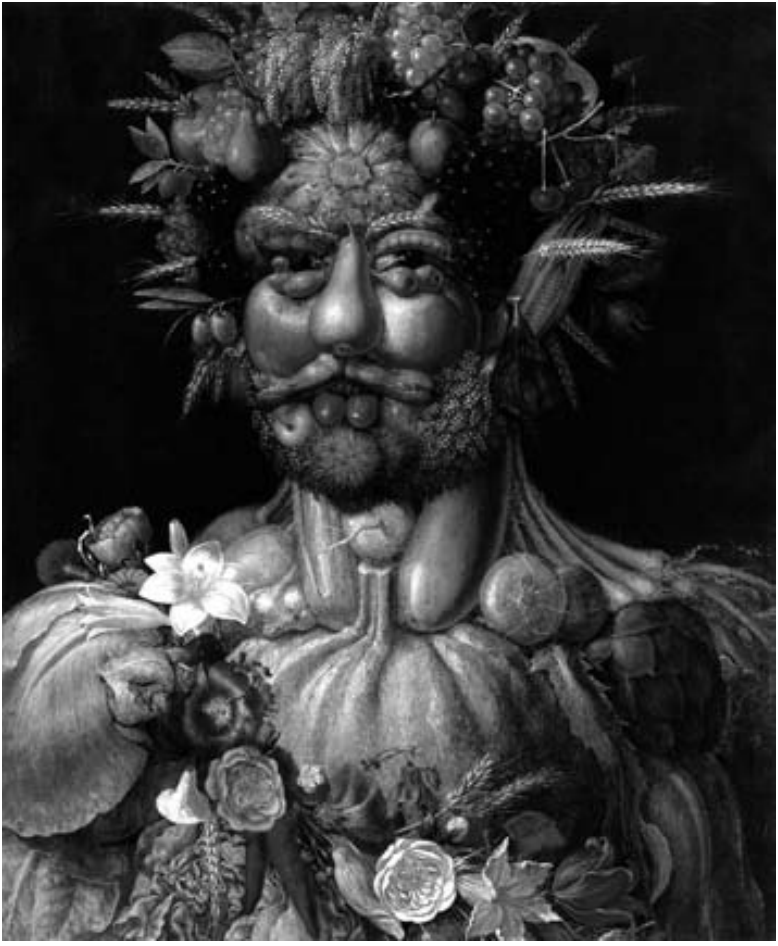
plusieurs attitudes. Dans la pratique d'historiens " positivistes », l'écrit étant le fondement de la science historique, le portrait scripturaire ou littéraire pouvait être un document digne des procédures savantes de l'analyse de texte. Quant au portrait figuré (dessin, gravure, peinture), il relevait de l'histoire de l'art, science auxiliaire de l'histoire, venant en illustration de ce qui était connu par ailleurs. Si on lui accordait quelque attention, c'était pour en faire une lecture psychologisante, relevant de la physiognomonie et révélant le caractère du personnage. Cette approche extérieure faisait preuve de naïveté, accordant un crédit indiscutable au représenté. Seul le portrait-charge et la caricature, par leur caractère outrancier et leur écart au réel, étaient crédités d'une efficace propre et échappaient donc au statut passif d'illustration. Les planches de l'Histoire de France illustrée depuis les origines jusqu'à la Révolution d'Ernest Lavisse, ouvrage paru en 1911, reflètent cette problématique ${ }^{4}$.

Depuis la révolution épistémologique de I'histoire dans les années 1960 et 1970, la production iconographique, et donc le portait, a gagné un nouveau statut, celui de document. La question posée alors fut celle de la vérité, en l'occurrence de la ressemblance. L'analyse portait sur la crédibilité à lui accorder et sur la valeur des informations contenues dans l'image. Un véritable discours de la méthode a été élaboré, notamment par Laurent Gervereau et Annie Duprat, assez semblable par ses protocoles à celui développé par les historiens positivistes sur le document écrit ${ }^{5}$.

Une autre attitude a vu le jour, par exemple lors des séminaires /conographie, propagande et légitimation dans le cadre de l'enquête internationale sur les origines de l'État moderne en Europe menée entre 1989 et $1992^{6}$. Cette approche, pas vraiment différente de la précédente mais un peu en décalage, consiste à voir le portrait comme un outil plutôt que comme un document. C'est dans ce cadre que Peter Burke pouvait parler de «fabrication » de Louis XIV ${ }^{7}$. Quittant la posture d'extériorité, de distanciation, d'objectivité (d'objectivation ?), le portrait peut être 
2. Piero della Francesca, Double portrait de Battista Sforza et de Federico da Montefeltro (recto) et Le Triomphe de la chasteté (verso), 1465-1466, Florence, Galleria degli Uffizi.
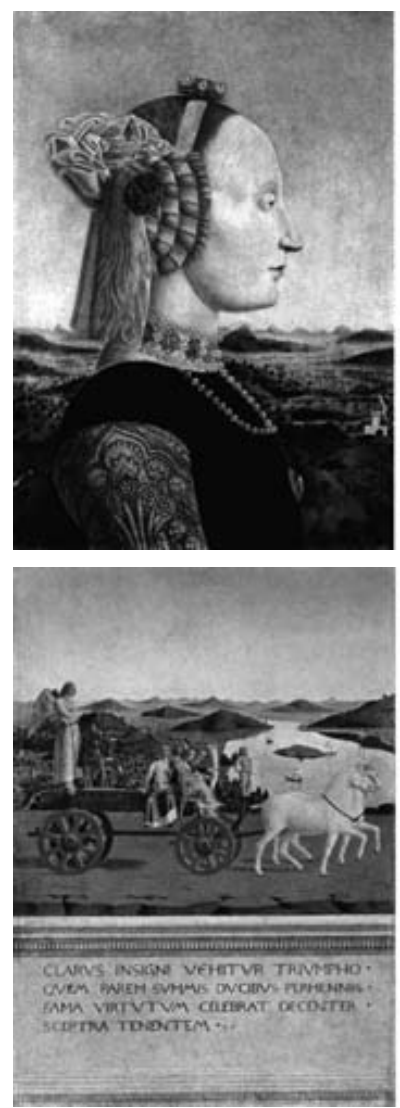

considéré, pour ainsi dire, de l'intérieur. La question posée n'est pas alors celle de sa vérité mais de son fonctionnement, de son efficacité et de son pouvoir. Ainsi, les éventuels " écarts " au réel ne sont pas disqualifiés mais sont interprétés en termes de stratégie. Cette posture de l'historien doit beaucoup à l'apport de la sémiologie, des sciences politiques et de la communication.

Antonio Pinelli. Selon moi, seules les approches relevant de l'histoire, de l'anthropologie et de la sémiologie, qui respectent le statut historique et artistique des portraits royaux, sont d'une extrême utilité. Respecter ce statut signifie prendre en considération leur nature de document visuel à l'aide des instruments d'analyse susceptibles d'en relativiser les sens et non pas de les absolutiser. L'image artistique possède une particularité : se présentant comme un témoignage du passé, elle vit cependant dans un "éternel présent », en proie à nos projections et à nos manipulations. Je me méfie des approches qui, profitant de cette vulnérabilité, soustraient l'œuvre d'art à la relativité de l'histoire et la projettent en une dimension extratemporelle, constituée d'archétypes et d'invariances. Pour citer un exemple concret, prenons le cas du célèbre Double portrait de Battista Sforza et de Federico da Montefeltro de Piero della Francesca, dont le verso intitulé Le triomphe de la chasteté, présente le duc et la duchesse sur des chars (1472, Florence, Galleria degli Uffizi ; fig. 2). Il est évident que l'analyse de ce double portrait n'exclut pas, et peut même mettre à profit, I'apport de disciplines comme l'histoire et l'anthropologie ${ }^{8}$. Toutefois, pour en comprendre pleinement les messages, il est nécessaire avant tout d'utiliser des instru-

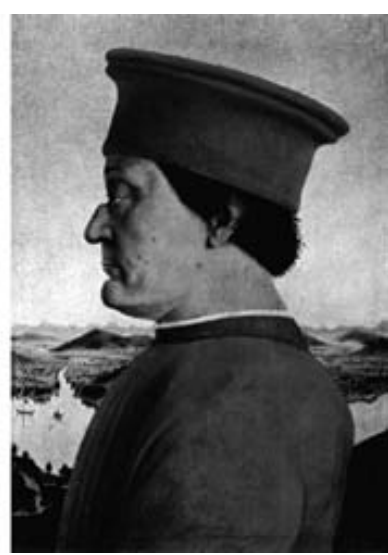
ments spécifiques à l'histoire de l'art comme l'iconographie, l'étude des techniques de représentation, etc. Dans ce cas précis, ils permettent de mettre en évidence des ressemblances avec le portrait royal, comme I'importance du modèle de la monnaie antique, sur laquelle les empereurs étaient représentés de profil au recto, tandis que figurait au verso une allégorie exaltant leurs vertus individuelles. En outre, l'œuvre illustre la diffusion, aussi bien dans le domaine des arts figuratifs que dans celui des apparats éphémères, du char de triomphe conçu comme une allégorie glorifiant les vertus morales, à l'image des Triomphes de Pétrarque. La maîtrise de la perspective et de l'éclairage par Della Francesca, et notamment l'interaction entre les personnages du premier plan et le paysage, contribue à l'idée d'une représentation géographique et allégorique des possessions du couple ducal.

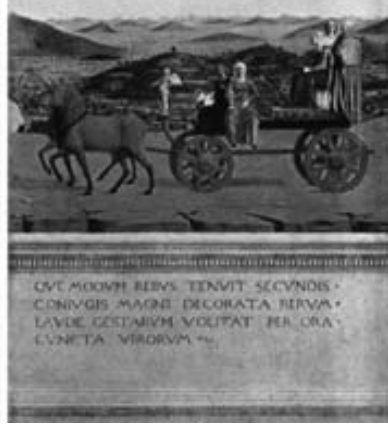

Barbara Stollberg-Rilinger. Grâce au tournant culturaliste de ces dernières décennies, le portrait du souverain est progressivement devenu l'objet d'une recherche interdisciplinaire. Cette évolution a rapproché les différentes sciences humaines et sociales, et a assoupli les frontières entre disciplines. La compréhension globale de la culture comme un système collectif de significations, continuellement recréé par ses discours performatifs, a déplacé le centre d'intérêt des contenus vers 
la manière dont ils sont produits. Cela vaut, à mon avis, pour l'histoire politique aussi bien que pour l'histoire de l'art. La fréquentation des mêmes auteurs classiques de l'anthropologie culturelle, et de la théorie des médias et des symboles - de Ferdinand de Saussure et Claude Lévi-Strauss à Jacques Derrida, Clifford Geertz et Umberto Eco, entre autres - a rapproché les deux disciplines. Les historiens de l'art ont commencé à s'occuper plus intensivement de la dimension communicationnelle des œuvres d'art et des stratégies de production liées à leur support ; les historiens politiques ont découvert que le pouvoir est toujours l'objet d'une construction symbolique et passe par la perception sensible. Avant tout, les historiens politiques ont commencé à s'intéresser aux images, considérées non plus seulement comme des illustrations de faits historiques mais comme des objets historiques sui generis.

La notion d'image est cependant ambiguë, et les représentants des différentes disciplines comprennent dans ce terme des choses différentes. En allemand, Bild signifie à la fois l'artefact artistique au sens étroit (pictura), l'image-reflet au sens large (imago), la représentation mentale (imaginatio) et enfin les images langagières, c'est-à-dire les métaphores et les récits. Quand les historiens de l'art parlent du portrait du roi, ils prennent généralement l'image au premier sens, tandis que les historiens l'entendent généralement dans un sens plus large. Or il me semble justement intéressant d'examiner les images matérielles et mentales dans leur interconnexion et dans leurs contextes communicationnels respectifs. C'est ainsi que, dans mon propre travail, j'étudie les échanges entre l'image, l'écrit et le rituel. Il s'agit de rendre à nouveau visible, dans un contexte historiquement donné, un ensemble de faits qui a été largement oublié en raison de la différenciation des sciences humaines ${ }^{9}$.

Diane Bodart. Les études de Louis Marin ${ }^{10}$ ont été un jalon fondamental pour la compréhension du portrait royal en tant que re-présentation : il possède des pouvoirs de substitution, d'intensification et de légitimation, qui résident aussi bien dans l'essence ontologique de l'image que dans les effets de sa manifestation. À votre sens, cette grille d'interprétation, forgée sur l'exemple de Louis XIV, est-elle plus généralement opératoire pour la représentation de tout souverain? Qu'en est-il de la valeur de présence du portrait du roi eu égard au pouvoir d'illusion de l'œuvre d'art?

Christine Tauber. Depuis les débuts de la théorisation du portrait, c'est sa capacité à rendre présent ce qui est absent, au service de la Memoria, qui a préoccupé les esprits. Cette fonction représentative et mémorielle est déjà tangible dans le mythe antique de sa naissance. Pline l'Ancien rapporte que la fille du potier Butadès de Sicyone était à tel point éprise d'un jeune homme qu'elle voulut absolument conserver son image lorsqu'il partit au loin, priant son père de réaliser un masque en terre cuite d'après l'ombre de son amant qu'elle avait dessinée sur une muraille. Si l'on accorde foi à cette légende antique, l'origine de l'art occidental dans son ensemble réside dans un portrait qui remplace l'amant absent. Leon Battista Alberti souligne au début du second livre du De pictura l'acquis principal de la peinture : sa qualité, semblable à la force de l'amitié, de rendre présent les absents et de rendre reconnaissables les défunts aux yeux des générations futures ${ }^{11}$. Dans le même temps, ce concept de représentation mémorielle permet un élargissement de l'utilisation politique du portrait. Saisir les traits principaux de la personne représentée signifie aussi se rendre maître de son être, et en posséder une image donne à son détenteur du pouvoir sur elle. Cette aspiration est visible dans la commande de Charles Quint à Titien de la série de portraits des vaincus de Mühlberg, dans laquelle le portrait de Charles Quint vainqueur figurait comme à la tête $d^{\prime}$ un cortège triomphal ${ }^{12}$. 
3. Giulio Paolini, Giovane che guarda Lorenzo Lotto, 1967, Laupheim, collection FER.

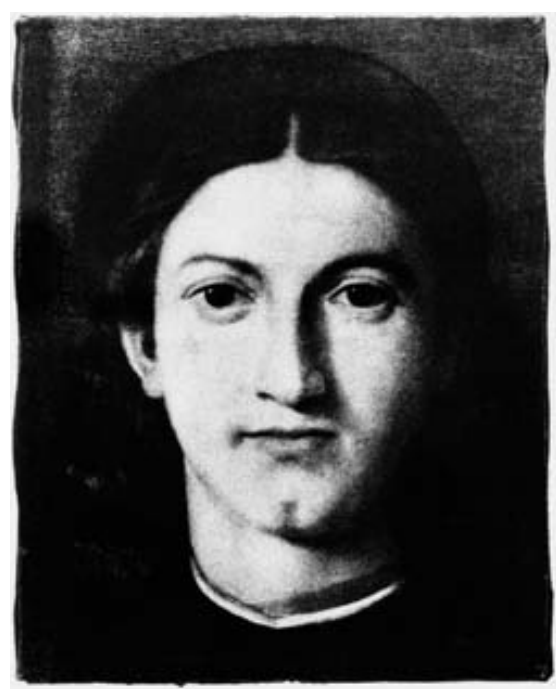

Mais ce serait sous-estimer la peinture de portrait à l'aube de la période moderne que de vouloir la réduire à une simple représentation magique de l'absent. Bien plus qu'une représentation symbolique, il s'agit d'un véritable projet artistique, réalisé grâce à des moyens esthétiques. Dans la grande galerie de Fontainebleau, des procédés d'allégorisation du portrait royal traduisent différents degrés de cryptage au moyen de signes hermétiques. Le roi y figure comme garant de la paix et fondateur de l'unité mais aussi - de manière plus ambiguë - comme ex utroque Caesar, représenté sur la dernière fresque en tant qu'interprète suprême des signes de sa galerie, faisant de celle-ci un portrait complet de sa puissance d'interprétation et de sa supériorité politique ${ }^{13}$.

Antonio Pinelli. Selon moi, le concept de re-présentation introduit par Marin ne concerne pas seulement le portrait du roi mais implique aussi une des fonctions constitutives de l'art, celle de rendre une absence éternellement présente. Ce n'est en effet pas un hasard si les Anciens ont raconté la naissance de la peinture et de la sculpture à travers l'histoire de la fille du potier de Corinthe, Butadès. Vaincre la mort, immobiliser le temps, cristalliser l'image de ce qui n'existe plus ou, inversement, représenter ce qui n'est pas encore présent ; depuis la nuit des temps, c'est précisément cette capacité qui a paré l'artiste d'une aura magique. Après avoir lu Le Portrait du roi de Marin, je me suis hâté de relire un traité sur l'imitation publié en 1823 par un auteur que j'ai beaucoup fréquenté, Quatremère de Quincy, dans lequel j'ai trouvé des assonances significatives, mais aussi des dissonances, avec ce que je lisais chez Marin. Voici ce qu'écrit Quatremère de Quincy à propos de l'image : "L'image n'est autre chose qu'une apparence de l'objet représenté. Il y a entre l'objet et son apparence toute la différence qui sépare ce qui est en effet de ce qui paraît être ; et ceci peut s'appliquer aussi à la ressemblance : celle qui appartient à l'image n'est autre chose qu'une apparence de ressemblance ${ }^{14}$. Quatremère de Quincy, qui a pourtant écrit des pages mémorables sur le pouvoir illusionniste de l'art, accentue beaucoup plus que Marin la distance qui sépare l'objet de sa représentation, au point d'en arriver à cette définition de l'art très lucide : "Oui, c'est précisément ce qu'il y a de fictif et d'incomplet dans chaque art, qui le constitue art. C'est de là qu'il tire sa principale vertu et l'effet de son action. $C^{\prime}$ est de là que vient le pouvoir de nous plaire ${ }^{15}$.

Dans le préambule de son traité, il se dit convaincu que ce sont les belles œuvres d'art qui engendrent les théories et non pas l'inverse, mais il fait une exception : "II y a des belles théories qui sont aussi en leur genre de beaux ouvrages, et auxquelles bien des personnes prennent plaisir ${ }^{16}$. À ce sujet, il serait intéressant de se pencher sur le lien entre les théories de Marin et la photographie de Giulio Paolini intitulée Jeune homme regardant Lorenzo Lotto (1967, Laupheim, collection FER ; fig. 3). Je ne crois pas que l'artiste italien puisse avoir exercé une quelconque influence sur les théories de Marin, mais elle évoque certaines de ses idées les plus géniales et les plus fécondes, comme celle du concept de l'opacité autoréflexive de l'image picturale. Comme dans le mythe de Narcisse, la représentation se reflète dans le regard de celui qui l'observe, tombe amoureuse d'elle-même et favorise un renversement du rapport entre l'image et le spectateur, entre le sujet et l'objet; dans le cas d'un portrait, il s'agit d'un renversement entre l'objet de la peinture - le visage du modèle - et le sujet de l'action picturale - l'artiste. 
Barbara Stollberg-Rilinger. L'étude sémiotique de Louis Marin, Le Portrait du roi, est loin d'avoir trouvé en Allemagne, du moins chez les historiens, l'écho qu'elle mérite ${ }^{17}$. L'ouvrage beaucoup moins ambitieux et plus lisible de Peter Burke sur la mise en scène du Roi-Soleil a exercé une influence considérablement plus grande ${ }^{18}$. Mais certains chercheurs allemands ont posé des questions similaires à celles de Marin, notamment dans la perspective des sciences juridiques, politiques, sociales et littéraires, la principale référence commune étant sans aucun doute l'ouvrage d'Ernst Kantorowicz, Les Deux Corps du roi ${ }^{19}$.

À partir de l'exemple de Louis XIV, le livre de Marin étudie les liens mutuels entre la représentation du pouvoir et le pouvoir de la représentation (une figure de style à la mode, dont ont joué d'innombrables titres publiés depuis). Marin souligne avant tout le lien entre la représentation politique et la représentation esthétique - deux dimensions du concept qui sont généralement étudiées par des disciplines différentes et sont donc toujours envisagées séparément. Tandis que la théorie politique et l'histoire s'occupent de la représentation comme procédé d'imputation politique, les études littéraires et artistiques visent la dimension esthético-symbolique de la représentation. En politique, «A représente $B$ » signifie : "Ce que fait $A$ vaut comme si $B$ I'avait fait ». Dans I'art, au contraire, " $A$ représente $B$ » signifie : " $A$ donne à voir $B$, il le rend perceptible aux sens », ou " $A$ incarne $B$ ». Marin thématise les rapports croisés, extrêmement complexes, qui s'établissent entre ces deux dimensions. L'essentiel à mes yeux est d'abord que, pour les acteurs du début de la période moderne, la représentation politique et la représentation symbolique, la production et la représentation d'une totalité politique, constituaient deux faces d'une même médaille. En outre, Marin postula que ce sont les actes de représentation qui, en un sens, produisent l'objet représenté - à savoir le corps politique.

Dans le contexte du Saint Empire romain germanique on trouve un type d'image spécifique qui se décline selon de nombreuses variantes : la représentation de l'empereur trônant parmi les princes électeurs (fig. 4) ${ }^{20}$. L'objet de ce type d'images est le rituel de l'Empereur siégeant in majestate, en grande pompe et apparat, un exercice démonstratif de souveraineté dont la forme cérémonielle précise se trouve décrite dans la Bulle d'or de 1356. Lorsque le roi ou l'empereur apparaissait ainsi dans le cercle des princes électeurs (ou de leurs représentants), ses faits avaient valeur d'actes formels de majesté et prenaient force de loi. Ainsi, la « séance en majesté » distinguait le roi en tant que personne naturelle de sa personne publique, porteur d'une persona et d'un rôle : c'était elle qui différenciait les deux corps du roi. Dans un tel rituel solennel, la majesté de l'empire et sa structure hiérarchique se manifestaient pour ainsi dire dans leur présence réelle. Ce qui s'accomplissait dans ces formes solennelles valait pars pro toto, comme si l'Empire tout entier en tant que corps politique l'avait réalisé.

Cette cérémonie présentait une qualité iconique. Les rituels sont en quelque sorte présentés et perçus comme des images vivantes dont le médium est les corps des acteurs. Les rituels partagent justement avec les images matérielles au sens strict cette "dynamique iconique » décrite par Gottfried Boehm comme " un surcroît intérieur propre aux images, qui se distingue de la surface du monde ${ }^{21}$. De même que les images, les rituels sont vus par les spectateurs comme une unité détachable de son environnement et se trouvent en tant que telle dotés de sens. Les rituels comme les images comportent en effet un geste déictique, et tous deux s'offrent au spectateur à l'intérieur d'un cadre - littéral ou métaphorique -, qui les
4. L'Empereur Charles IV et ses princes électeurs, Bulle d'or de 1400, Vienne, Österreichische Nationalbibliothek, Cod. 8070, fol. 2 r.

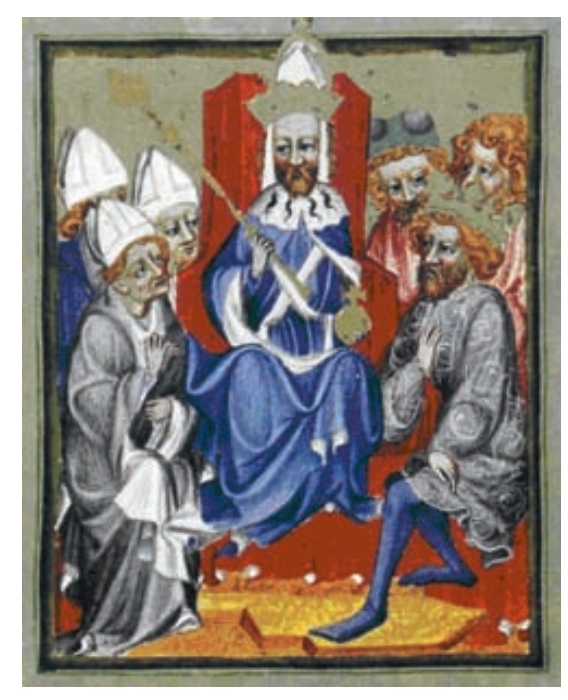


5. Louis Marin, Le Portrait du roi, Paris, 1981.

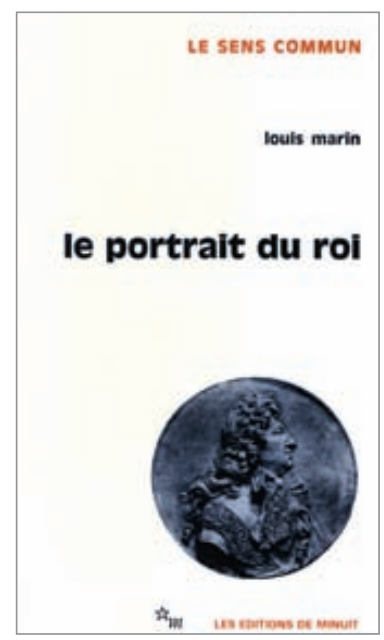

distingue de ce qui n'est pas rituel ou image. Cette différence iconique, cette démarcation démonstrative qui s'effectue par l'encadrement et qui caractérise aussi bien les actes rituels que les images matérielles, fait des rituels des sujets privilégiés de la représentation picturale.

L'efficacité iconique de la représentation du rituel de l'Empereur siégeant in majestate est attestée par la pérennité de son usage. Ce type d'image servit d'abord à illustrer la page de titre des recueils de lois, puis il apparut, jusqu'à la fin de l'Empire, sur des certificats de noblesse, des lettres de concession d'armoiries et d'autres documents de ce genre. La majestas imperialis se trouvait par la mise en scène comme source de légitimation et instance de sanction du droit. On continua à utiliser ce type d'image à chaque fois qu'il s'agissait d'instaurer une obligation au nom de l'Empire entier. La majesté de l'Empire s'incarnait encore dans ces représentations secondaires, alors que les rituels eux-mêmes, c'est-à-dire les formes de représentation réelles ou de premier degré, étaient depuis longtemps tombés en obsolescence.

Gérard Sabatier. Pour répondre à la question de la valeur de présence du portrait du roi eu égard au pouvoir d'illusion de l'œuvre d'art, on pourrait citer Marin lui-même : « la représentation est l'opération qui met la force en réserve dans les signes, en transformant la force en pouvoir. Le résultat de cette opération substitutive est un effet de croyance : celui qui voit le portrait équestre du roi croira que le souverain dispose d'une force sans limites [...] Ainsi le roi n'est-il pleinement roi que dans la représentation [...] le pouvoir, $c^{\prime}$ est la représentation ${ }^{22}$. Le promeneur de la place Vendôme en 1699 croyait-il que Louis XIV disposait d'une force sans limites? Si le sémiologue se place du côté des signes, donc de l'émetteur, l'historien est attentif au dialogue effectif entre l'émetteur et le récepteur, et pourra avoir quelque doute, dans ce cas particulier. Il peut toutefois adhérer à la problématique de l'effet de croyance.

Pour l'historien, Louis Marin a permis de comprendre le rôle que jouait le portrait dans une phase spécifique de l'histoire du pouvoir : l'état princier de la première modernité dans l'Europe chrétienne. D'un élément considéré comme accessoire, bien qu'important pour sa valeur esthétique ou mémorielle, il a fait un outil fondamental au service de stratégies d'émergence, de distanciation et d'appropriation. Comme le rappelle Diane Bodart dans sa question, le pouvoir du portrait royal décrit par Marin (fig. 5) s'établit selon différentes modalités. En premier lieu, I'intensification, marquée par la monstration de la force, par la production d'images - ou plutôt d'imaginaires - dont le maximalisme (les portraits avec une identification aux dieux et aux héros antiques par exemple) était souvent inversement proportionnel à la puissance effective $^{23}$. En second lieu, la substitution, la sédentarité du prince et la possession de vastes territoires rendant en effet nécessaire le marquage de l'espace approprié. Enfin la légitimation, car dans la monarchie de droit divin, avec ou sans sacre, la relation des sujets au roi se faisait sur le mode religieux. Dans I'Italie espagnole ou dans la France de Louis XIV, le portrait du roi assurait effectivement la médiation du sujet avec le souverain, comme le faisaient les images saintes dans le catholicisme tridentin. En 1687, par exemple, les actions de grâce pour la guérison de Louis XIV recoururent au protocole cultuel du portrait ou de la statue environnés de cierges ${ }^{24}$.

L'opération, pour fonctionner parfaitement, nécessite cependant chez les destinataires des conditions de réceptivité adéquates. Qu'en était-il dans le monde protestant, où le rapport à l'image était différent ? Chez les princes allemands luthériens, la force opératoire du modèle catholique du portrait entraîna vite une adhésion qui vint contredire le radicalisme des origines du protestantisme, auquel les calvinistes et 


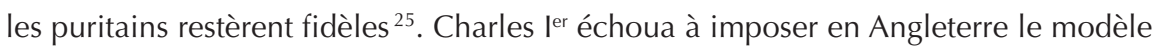
français. Le janséniste Pascal pose bien le primat de la réception avec sa fable des gens qui voulaient un roi : " Un homme est jeté par la tempête dans une île inconnue, dont les habitants étaient en peine de trouver leur roi qui s'était perdu; et ayant beaucoup de ressemblance de corps et de visage avec ce roi il est pris pour lui et reconnu en cette qualité par tout ce peuple ${ }^{26}$. Rapportée par Louis Marin, elle révèle le désir du peuple qui fit reconnaître le naufragé en tant que roi, alors que ce dernier savait qu'il n'était qu'un homme comme eux.

Diane Bodart. Dans quelle mesure la théorie des deux corps du roi étudiée par Ernst Kantorowicz, au-delà de la fiction juridique propre à certaines monarchies, peutelle servir dans une acception plus large à l'analyse du portrait royal ? En d'autres termes, l'idée de la double nature du souverain - individuelle et politique - détermine-t-elle sa représentation? Peut-on parler d'un portrait privé et d'un portrait public du prince?

Antonio Pinelli. Je répondrai sans hésiter par l'affirmative. La théorie des deux corps du roi peut servir à analyser les portraits des monarques, et je crois que l'on peut identifier une ligne de fracture très nette entre l'effigie privée et l'effigie publique d'un souverain. La continuité du corps politique du roi et sa perpétuation d'un monarque à l'autre donnent lieu à une iconographie plus ou moins stable du portrait d'État. Généralement, il prend place également dans une série, s'inscrivant dans une galerie idéale où les traits individuels changeants se fondent dans l'uniformité iconographique de ladite série. Un exemple concret et révélateur est celui des portraits des papes. Les pontifes, comme on le sait, sont des souverains très particuliers puisqu'ils sont élus et ne forment pas de dynastie. Toutefois, la théorie du double corps vaut pour eux aussi, d'autant plus qu'ils légitiment leur pouvoir en qualité de vicaires terrestres du Christ, dont la double nature, divine et humaine, est à l'origine de cette théorie. Le népotisme est la stratégie spécifique mise en œuvre par les papes pour perpétuer leur pouvoir à travers une forme voilée de " dynastisation ». Le portrait du pape Eugène IV de Jean Fouquet, aujourd'hui perdu, nous est connu grâce à une gravure tardive qui montre le pontife seul (fig. 6), alors que dans l'œuvre de Fouquet il était accompagné de ses deux neveux ; une présence indiquée par les sources et confirmée par le Portrait de Léon X avec ses deux neveux cardinaux de Raphaël (1518-1519, Florence, Galleria degli Uffizi; fig. 7), qui s'inspire clairement du portrait pontifical perdu. Après cette période de népotisme affiché et triomphant, dont l'exemple le plus éloquent est offert par la fresque de Melozzo à la Biblioteca Apostolica Vaticana (1477, Rome, Biblioteca Apostolica Vaticana), un nouveau paradigme s'établit, dont relève notamment la version gravée du portrait d'Eugène IV de Fouquet, qui n'admet pas de présences étrangères aux côtés de la figure du pape. Ce changement de conception

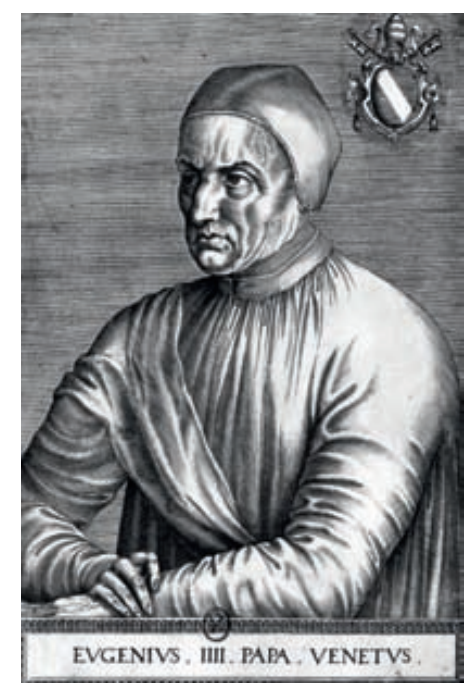

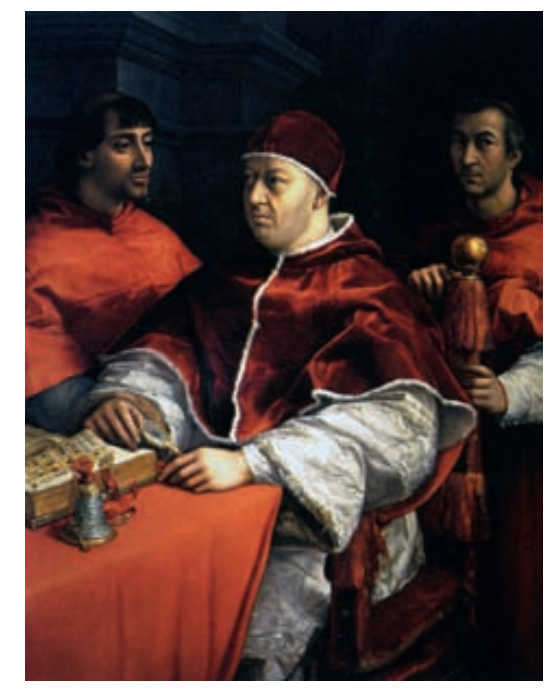

6. Gravure d'après Jean Fouquet, Portrait du pape Eugène IV, 1568, Paris, Bibliothèque nationale de France.

7. Raphaël, Portrait de Léon X avec ses deux neveux cardinaux, 1518-1519, Florence, Galleria degli Uffizi. 
8. Hyacinthe Rigaud, Louis XIV en costume de sacre, 1701, Paris, Musée du Louvre. explique la disparition des cardinaux neveux, qui servaient à l'origine de garde d'honneur du pape, prolongeant l'influence de son " corps politique » dans le temps.

Même en faisant abstraction de son livre le plus célèbre, Kantorowicz est selon moi l'un des principaux points de repère méthodologiques pour qui voudrait analyser en profondeur la spécificité des arts figuratifs en tant que témoignages historiques. Comme l'a reconnu Erwin Panofsky, il a développé en effet une méthode capable de combiner l'étude des textes et des documents à celle de la sculpture, de la peinture, de l'illustration et de la numismatique, de manière à traiter le matériau figuratif comme objet d'étude sui iuris et non pas comme un ensemble de témoignages complémentaires. Malheureusement, ce type d'approche est encore loin d'aller de soi. Il est certes indéniable que l'attention croissante accordée à la dimension matérielle, psychologique et esthétique de l'existence s'est traduite, dans les études historiques de ces dernières décennies, par un recours massif aux images, conçues comme un réservoir précieux de traces du passé ${ }^{27}$. Il est néanmoins tout aussi vrai que de nombreux historiens se fient à la notion empirique, ô combien trompeuse, selon laquelle l'image parle d'elle-même, et qu'il reste parmi eux de fortes réticences à accorder aux témoignages figuratifs le même degré de dignité qu'aux témoignages verbaux. Montrer que les témoignages figuratifs ont leur propre statut et qu'ils doivent être lus au moyen d'instruments susceptibles d'en déchiffrer les significations est une entreprise qui reste en grande partie à accomplir. La faute ne revient pas tant aux historiens qu'à nous, historiens de l'art, car nous sommes encore peu conscients des immenses potentialités herméneutiques que pourrait receler un appareil méthodologique plus riche et plus mûr, capable d'amorcer une refondation épistémologique de notre discipline, finalement assez jeune.

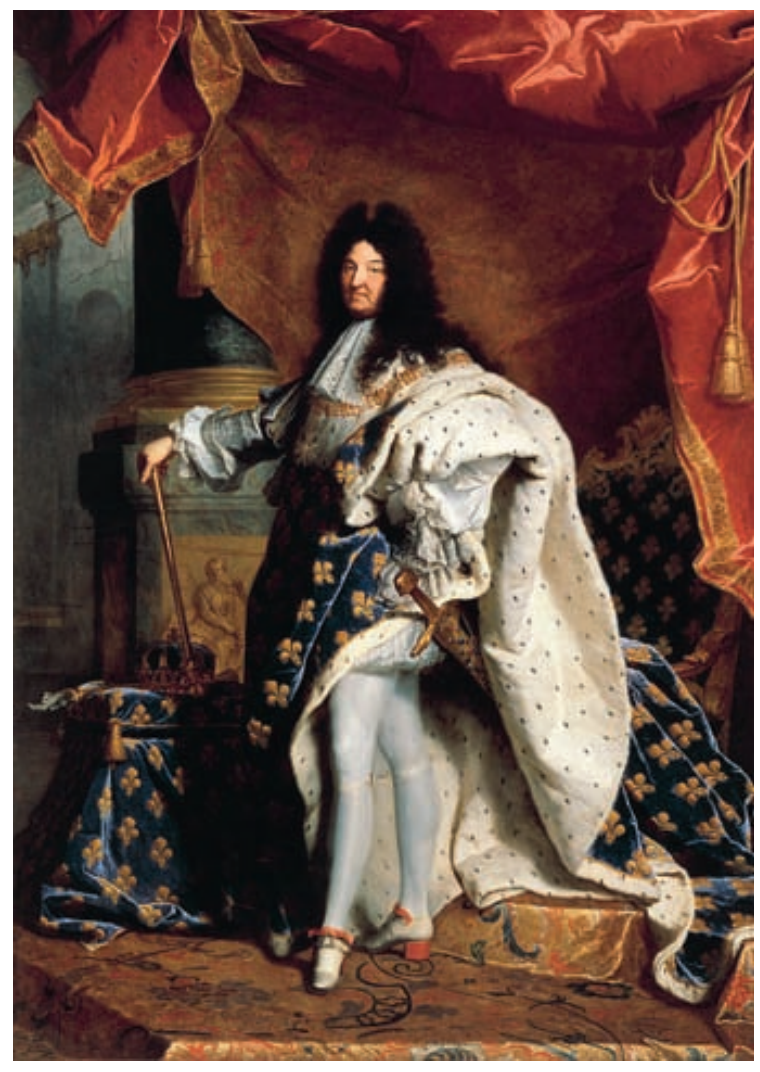

Gérard Sabatier. Bien avant Kantorowicz, les théoriciens du portrait italiens et espagnols avaient reconnu la double nature du corps du roi : "Les princes chrétiens [...] revêtent en même temps deux personnes, l'une publique et l'autre privée », écrivait Paleotti en $1582^{28}$. Les portraits des souverains (et des membres de leur famille, car la dignité est une affaire de sang) se devaient donc de rendre visible la ressemblance physique et la convenance au statut politique. C'est en fonction de cette double exigence que doit être conduite l'analyse du portrait royal, tout en appréciant les phases de son élaboration, et en interprétant l'accueil du commanditaire et du public. Ainsi, les postures « au naturel » du roi esquissées par Charles Le Brun pour l'escalier des Ambassadeurs à Versailles furent-elles rectifiées sur les peintures montrant un corps maîtrisé. Selon la même logique, la statue équestre de Louis XIV par Bernin fut disqualifiée comme portrait du roi au profit de celui de François Girardon, infiniment moins séduisant mais conforme à l'idée de souverain. Louis XIV en costume de sacre de Hyacinthe Rigaud (1701, Paris, Musée du Louvre ; fig. 8) exhibe tous les ingrédients du corps politique, mais ses jambes de danseur et son visage d'homme de 67 ans appartiennent au corps physique. Les portraits de Philippe IV de Diego Velázquez, tout en noir et sans aucun regalia (1624-1627, 
Madrid, Museo del Prado ; fig. 9), expriment par leur seul physique le statut monarchique du sujet ${ }^{29}$. Soulignons en outre que le décorum n'est curieusement pas un critère pour signifier l'une ou l'autre nature du corps du roi.

La question du portrait privé ou public a-t-elle à voir avec le double corps? Autrement dit, peut-on avoir un portrait privé qui soit celui du seul corps physique, l'ostentation des deux corps caractérisant le seul portrait public ? En fonction des codes de représentation, mais aussi en raison de l'adhésion à l'idéologie monarchique qui reconnaît la spécificité du corps du prince, un portrait du roi comprend toujours une combinatoire des deux corps, à quelqu'étape de sa vie et en quelque circonstance qu'on le représente. On ne peut parler de portrait privé ou public du prince, mais seulement d'usage privé ou public. Dès le XVII siècle, la vogue grandissante du portrait dans l'aristocratie comme dans la bourgeoisie a "désidéologisé » le genre ; l'exigence de ressemblance physique prima sans que disparaisse la nécessaire monstration du statut social. Cette " démocratisation » du portrait - loin du droit à l'image des théoriciens du XVI siècle réservant le portrait aux seuls souverains - a influencé la conception du portrait du prince au bénéfice de son corps physique. Peut-on parler de désacralisation? Au XIX ${ }^{e}$ siècle, quand le pouvoir fut partagé entre le souverain et les assemblées, il devint nécessaire de pourvoir son portrait d'insignes royaux, sa seule apparence ne suffisait plus à exprimer la royauté. Le portait n'en resta pas moins officiel, mais il congédia la notion d'incarnation. La monarchie ne fut alors plus un état mais une fonction extérieure à celui qui l'exerce, lequel ne fut plus un roi, mais un simulacre.

Christine Tauber. Il est possible, sans recourir à la thèse un peu grossière de Kantorowicz, d'élaborer un double usage de l'image d'après la manière dont on concevait le portrait du monarque à l'aube de la période moderne. Par exemple, l'analyse du contexte fonctionnel et usuel des images dans le cadre de l'autoreprésentation royale à la cour de François I ${ }^{\text {er }}$ permet de faire la différence entre un portrait conçu et réalisé à des fins officielles et une effigie destinée à une élite plus restreinte, deux conceptions qui me semblent plus opératoires que celles de «public » et "privé ». La décision de faire venir les maniéristes italiens à Fontainebleau correspondait à une nouvelle orientation dans la politique artistique royale : la maniera, envisagée $d^{\prime}$ un point de vue très moderne, prit la place d'une tradition nationale rassurante ${ }^{30}$. Les peintres du Nord, quant à eux, conservèrent la tâche artistique tout à fait spécifique de réaliser des portraits, dont le roi étendit la conception, après 1525, à une véritable politique. Contrairement au peintre de cour typique de la Renaissance qui devait être versé dans tous les domaines artistiques, Jean Clouet a dessiné et peint exclusivement des portraits, et a gagné ainsi (comme Appelle, et plus tard François Clouet, son fils) le monopole du portrait royal. Cette expertise professionnelle permit au faciès du roi, en soi unique, de rester toujours identique à lui-même et garantit ainsi la continuité de l'exercice du pouvoir légitimé iconographiquement (après 1526, Paris, Musée du Louvre ; fig. 10) ${ }^{31}$. Mais dans I'utilisation de la reproduction du visage royal, une stratégie de la raréfaction fut poursuivie dès le départ : les dessins de Clouet, prévus d'abord comme des études préparatoires aux portraits à réaliser, ne furent reproduits qu'à la main, le passage à une reproduction imprimée pour agir sur les masses n'ayant pas été prudemment franchi. Chaque copie était vraisemblablement visée par I'atelier de Clouet, de sorte que seules des reproductions autorisées furent mises en circulation. Le roi resta ainsi maître de ses portraits et de leur diffusion, qu'il limita de manière élitiste au cercle restreint de 

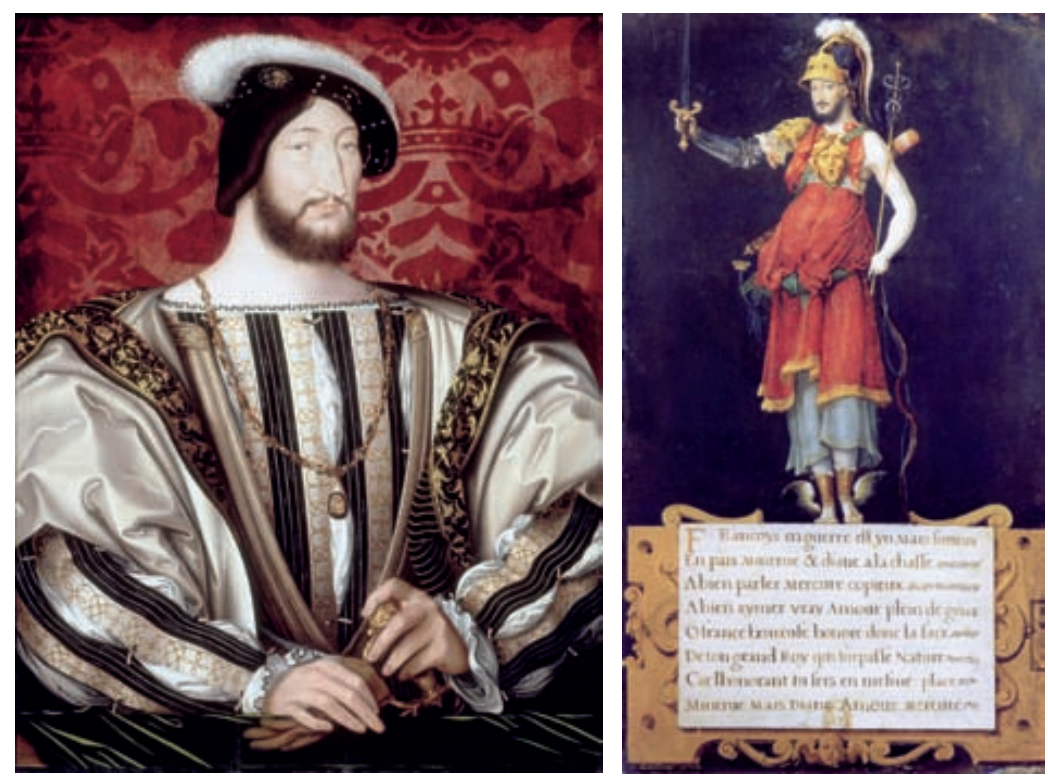

la société de cour et au commerce diplomatique. Le portrait composite du roi ${ }^{32}$, vers 1532-1537 et longtemps attribué à Niccolò Belin de Modène, est I'un des plus hermétiques (Paris, Bibliothèque nationale de France ; fig. 11) ; Panofsky le qualifia $d^{\prime}$ " hybride monstrueux $»^{33}$ et nous n'avons pas de renseignements précis sur sa fonction. Dans un acte autonome $d^{\prime}$ appropriation éclectique, les dieux y sont fragmentés et détrônés. L'Olympe tout entier est mis en pièces et, selon ses désirs et les impératifs de représentation, le roi est figuré dans ses attributs comme un « surdieu » (Übergott) dominateur qui dispose de toute évidence d'une légitimité in-

10. Jean Clouet, Portrait de François ler, roi de France (1494-1547), après 1526, Paris, Musée du Louvre.

11. Anonyme (attribué à Niccolò Belin da Modena), Portrait allégorique de François ${ }^{\text {er }}$, vers 1532-1537, Paris, Bibliothèque nationale de France. contestable. Le monopole de la représentation est ici encore maintenu, car on ne peut reconnaître le roi que par l'introduction du type de physionomie créé par les Clouet.

Barbara Stollberg-Rilinger. Au-delà de la théorie des deux corps, le célèbre livre de Kantorowicz traite d'un autre sujet : il montre comment les savants médiévaux ont commencé à développer une idée abstraite du pouvoir comme institution - ce qui n'allait nullement de soi. Ils sont parvenus à le faire grâce à la double compréhension du corps, en distinguant entre le corps naturel, mortel, et le corps supra-personnel, éternel et en quelque sorte institutionnel. Il nous est difficile aujourd'hui de suivre ce cheminement, car nous sommes accoutumés à toujours penser par abstractions. Nous rencontrons donc le problème inverse : nous n'arrivons pas à comprendre le processus de formation d'institutions abstraites comme l'État, ni les pratiques, les représentations ou les objectivations concrètes dont il est issu. Le cultural turn nous a aidés dans cette voie, et la redécouverte du livre de Kantorowicz compte sans aucun doute parmi les déclencheurs principaux de ce tournant.

En ce qui concerne la différence entre le portrait privé et le portrait officiel du roi, il me semble d'une part que le portrait privé est un phénomène plus tardif, qui n'apparaît qu'au XVIII ${ }^{e}$ siècle, et d'autre part qu'il ne faut pas confondre la distinction entre le portrait officiel et le portrait privé du souverain avec la distinction entre le corps naturel et le corps institutionnel au sens de Kantorowicz. Certes, il s'agit dans les deux cas d'un processus de différenciation, d'une distinction de rôles. Mais le corps naturel du roi au Moyen Âge n'est jamais un corps privé et toujours un corps officiel. La mise en scène du souverain comme personne privée au XVIII ${ }^{\mathrm{e}}$ siècle suppose une différenciation sociale avancée, un sens développé de l'intimité familiale, un espace privé protégé, toutes choses qui à ma connaissance n'existaient pas à au début de l'époque moderne. Autrement dit, la distinction entre privé et officiel ne correspond aucunement à la différence entre naturel et institutionnel. 
Diane Bodart. La notion contemporaine de propagande, dont l'emploi pour l'Ancien Régime n'a pas été sans soulever de débats méthodologiques, est communément associée à l'étude de l'image royale pour rendre compte des stratégies figuratives du pouvoir monarchique. Compte tenu de votre démarche, cet anachronisme assumé vous paraitt-il éclairant pour appréhender les enjeux et les usages du portrait du roi ?

Gérard Sabatier. On entend par propagande une stratégie de communication visant à convaincre. Impliquant la mise en œuvre de techniques spécifiques et l'existence d'une opinion publique, on peut contester sa pertinence pour les époques qui ignoraient les unes comme l'autre. Cependant, considérée comme un " anachronisme assumé ", c'est-à-dire en refusant de la transférer telle quelle mais en accordant de l'attention à ses prérequis, la notion de propagande peut être éclairante pour appréhender les enjeux et les usages du portrait du roi.

Il faut considérer chaque cas comme particulier et lui appliquer une grille d'analyse systématique. La partie se joue à deux, émetteur et récepteur, avec un enjeu : le message à faire passer, en l'occurrence la signification du portrait. II faut prendre en compte la localisation (lieu privé ou public), le statut des publics, le dispositif de présentation et la visibilité de l'œuvre. Dans le cas du portrait multiple, la modalité de reproduction (répliques, gravures...), ainsi que le tirage et le coût, et donc le statut social des acquéreurs, rentrent également en ligne de compte. Quant à la compréhension des œuvres, les procédés figuratifs (réalisme, mythologie, allégorie, souvent en composition) nécessitent pour être appréhendés une culture des signes que nous n'avons plus, qui pouvait s'opérer d'une façon ségrégative (suivant des divisions culturelles et sociales) mais qui comportait une vulgate sémantique assez commune.

Il y a les portraits sans public, trop haut, trop loin, trop sombre, en contrejour (au plafond de l'escalier de l'Escorial, ou de celui des Ambassadeurs à Versailles). Leur fonction est exaltative, mémorielle, de l'ordre de la louange. Aussi inaccessibles sont les portraits réservés au prince, à ses intimes et à ses compétiteurs, à l'image des peintures de manuscrits ${ }^{34}$ ou celles de la galerie François ler à Fontainebleau ${ }^{35}$, assimilables à des " hiéroglyphes ", des images cryptées accessibles (à tous les sens du terme) au seul prince, ainsi institué maître des savoirs. Inversement, la lisibilité et la publicité maximales, dont jouissent par exemple les monnaies, les tableaux, les statues et les bas-reliefs dans les bâtiments officiels ou à leurs façades, ou les monuments équestres sur les places royales, sont-elles les signes d'une propagande ? Ces portraits, toujours sur le mode triomphal, ne visent pas à convaincre de l'excellence du prince mais énoncent un imaginaire monarchique de convenance. Par ailleurs, ils ne fonctionnent pas de manière autonome, mais dans leur relation avec l'objet (la pièce de monnaie) ou le lieu (le bâtiment ou la place) où ils sont apposés, devenant des marqueurs d'identité (monnaie "royale »), et d'appartenance (espace du royaume). Ce sont des sceaux, non des médias.

Est-ce à dire que le portrait royal n'est jamais propagande ? Il l'est lorsqu'il s'agit de convaincre une opinion réticente de la légitimité d'Henri IV par exemple, ou de la guerre contre l'Espagne. La monarchie française recourt alors au décorum des entrées royales et à un média de masse, la gravure, le vocabulaire héroïque ou mythologique n'étant pas un obstacle dans le contexte culturel du temps ${ }^{36}$. D'autres cas sont plus complexes, comme la Galerie des glaces à Versailles, un lieu ouvert où se pressait le public. Son vocabulaire héroïque a été simplifié, et des livrets d'explication étaient à la disposition des visiteurs. Dans les peintures de la voûte, le portrait du roi appartient indiscutablement au genre de la convenance, mais en se positionnant en opposition aux puissances hostiles à la France, il ouvre une polémique s'inscrivant 
dans un processus de propagande. La représentation du roi à la romaine est celle de tous les princes depuis presque deux siècles, mais il est seul à être identifié par son visage propre ; les " puissances » ennemies sont figurées par des allégories de I'Allemagne, de l'Espagne et de la Hollande, comme si leurs souverains ou dirigeants, insignifiants au sens propre, ne méritaient pas leur portrait. Inversement, la figure de Louis XIV devient I'allégorie du roi : un corps d'autorité caractérisé par la centralité, l'immobilisme et l'impassibilité. En regard, gesticulant, mues par leurs passions, les «puissances » impuissantes et sans visage sont disqualifiées ${ }^{37}$. Ces différences de traitement et la valorisation outrageuse de Louis XIV furent bien perçues comme une propagande, comme en témoigna la réaction critique des étrangers ${ }^{38}$.

Christine Tauber. Le concept de propagande me semble peu approprié pour comprendre les stratégies de domination et de légitimation du pouvoir monarchique qui se manifestent dans le portrait royal. Étant donné que " propagande » au sens restreint caractérise une technique de racolage utilisée spécialement et de manière intentionnelle pour dénoter une mise sous influence systématique et une sécurisation de l'exercice du pouvoir visant à former certaines manières de voir, à manipuler des savoirs et à guider les comportements, ce concept n'a de sens que s'il est posé dans le contexte des idéologies totalitaires. Appliqué au portrait du roi, il court le risque de dégrader I'œuvre d'art en un outil idéologique de manipulation de l'opinion. À cet usage instrumentalisé de l'image, j'opposerais la notion d'une politique du portrait qui fait partie intégrante de la pratique du pouvoir monarchique. Certes, l'effigie du roi a été utilisée de manière très consciente pour produire une « image de marque » précise et voulue, mais cela eut lieu la plupart du temps grâce à de subtils moyens artistiques et non par une simple propagande. Si l'on considérait l'artiste comme un agent de propagande, il ne serait qu'un illustrateur d'idées. J'aimerais lui opposer un modèle qui ne décrit plus les rapports de patronage comme une forme d'instrumentalisation des artistes à des fins de représentation et de pouvoir ${ }^{39}$. Il s'agirait plutôt de questionner les préférences et les critères de sélection qui entrent en jeu lorsque les détenteurs de la puissance politique et des moyens économiques décident, en tant que commanditaires d'œuvres d'art (et dans notre cas de portraits), d'entrer dans une relation professionnelle avec des artistes. N'existe-t-il pas chez les artistes une sorte d'aristocratie intellectuelle qui, tout en s'appuyant sur la capacité à créer de manière autonome des représentations artistiques et symboliques, correspond à la noblesse de robe du roi ? Celui-ci ne dispose pas lui-même de ce pouvoir artistique, mais il en a grand besoin pour sa maîtrise du pouvoir et il en résulte un rapport de dépendance mutuelle entre le commanditaire et l'artiste. C'est donc un choix crucial d'engager un ou plusieurs artistes qui soient capables de fabriquer le capital symbolique dont le roi a tellement besoin pour montrer à tous la légitimité incontestée de son règne.

Antonio Pinelli. Concernant l'Ancien Régime, je préfère au terme " propagande » I'expression «message idéologique confié à une œuvre d'art ». S'ouvre alors tout un éventail de questions. Qui définit ces messages ? Qui et combien sont les acteurs en jeu? À quelles méprises ces messages sont-ils exposés ? Est-ce la seule iconographie qui véhicule le message de l'œuvre, ou bien aussi son style ? Autant de questions névralgiques que j'aborde entre autres dans I'introduction de mon livre La bellezza impura ${ }^{40}$.

Pour revenir à l'exemple des portraits pontificaux, déjà cités plus haut, et en particulier ceux du XVe siècle, il est intéressant de s'attarder sur celui que Martin V 
confia à Gentile da Fabriano, aujourd'hui perdu mais dont la composition nous est connue grâce à diverses sources du Quattrocento. Elles nous apprennent que Martin $\mathrm{V}$ avait voulu se faire portraiturer entouré du collège cardinalice au grand complet, qu'il avait composé avec une grande habileté géopolitique de manière à ne mécontenter aucune «nation » importante. En donnant au tableau un message idéologique, il réussit en une seule mise en scène l'équilibre entre les deux charges contradictoires qui lui avaient été confiées lors de son élection : réunifier sous son sceptre l'Église d'occident pour mettre fin au schisme qui la déchirait depuis des décennies, tout en acceptant le principe de collégialité du pouvoir pontifical, dont la doctrine conciliaire était partisane. En déplaçant le principe de collégialité conciliaire au sein du collège cardinalice et en montrant, à travers les portraits de chacun des cardinaux qu'il avait nommés, la sagacité géopolitique qui avait présidé à la composition dudit collège, Martin $V$ confia à l'éloquence visuelle du tableau de Gentile da Fabriano un message idéologique et politique tout autre que banal.

Enfin, la fresque de Melozzo, citée plus haut

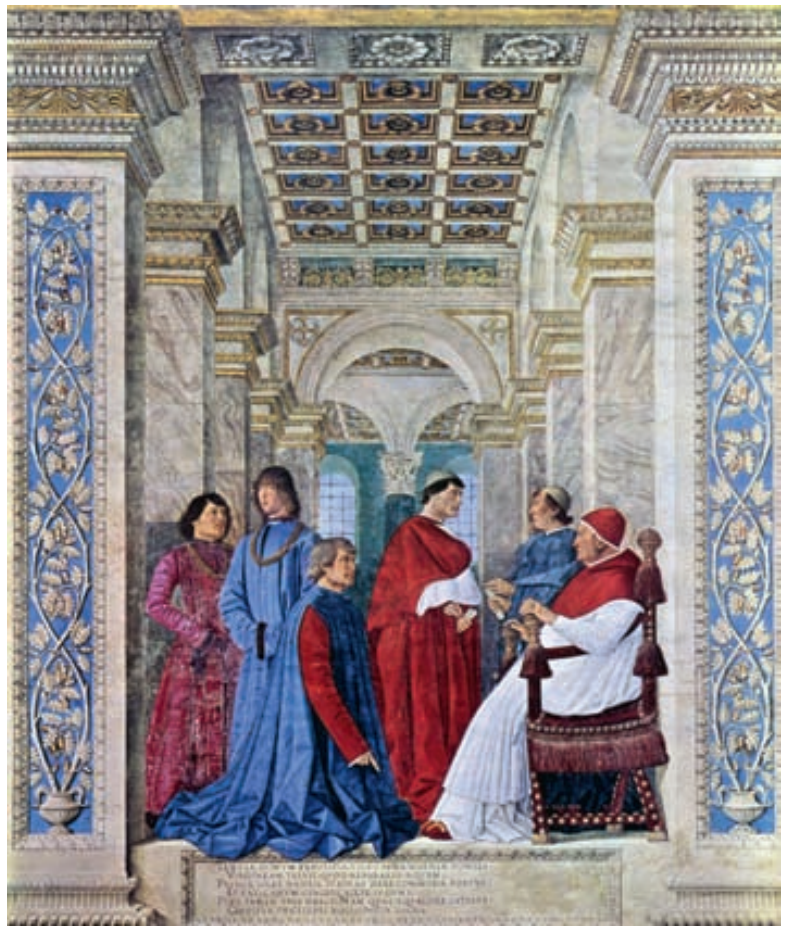
(fig. 12) permet d'illustrer certains des principaux éléments qu'il faut préciser lorsque l'on évalue une œuvre d'art présentant clairement un aspect propagandiste. Tout d'abord, il est nécessaire d'identifier son emplacement d'origine exact afin de reconnaître ses destinataires privilégiés (dans notre cas, respectivement, la salle de lecture de la Bibliothèque apostolique vaticane, et les laïcs et les clercs qui avaient accès à celle-ci). Il faut également savoir déchiffrer le véritable sujet de l'œuvre, en l'occurrence Sixte IV qui, en présence de ses neveux, deux laïcs et deux cardinaux, écoute les vers glorifiant ses réalisations dans le domaine architectural et artistique, dans une salle qui évoque la Bibliothèque apostolique vaticane rénovée ${ }^{41}$. Indissociable du sujet, le style de la fresque de Melozzo a aussi son importance. En disposant la scène selon l'optique d'un léger raccourci de bas en haut, pesant presque sur le spectateur, I'artiste a en effet rendu plus imposants les personnages représentés, renforçant aussi l'éloquence du message confié à l'œuvre.

Barbara Stollberg-Rilinger. L'usage que les souverains ont fait des arts visuels peut-il être décrit comme une " propagande » ou une stratégie politique ciblée ? D’un côté, sans aucun doute, oui. Le concept de propagande n'est nullement anachronique dans ce contexte, car il trouve ses racines, comme on le sait, dans la politique catholique de confessionnalisation. Les recherches récentes, par exemple de Naïma Ghermani (fig. 13) et de Dorothee Linnemann, ont éclairé de manière convaincante les stratégies de production auxquelles répondaient les portraits de souverains, mais aussi de diplomates ${ }^{42}$.

D'un autre côté, il faut émettre deux sortes de réserves. À aucune époque les souverains n'ont été seuls maîtres de la politique de l'image ; ils n'ont jamais pu contrôler parfaitement l'usage qui était fait de leur image dans l'espace public. Cette situation ne date pas seulement du XVIII siècle, au moment où le public devient " critique » en un

12. Melozzo de Forlì, Le pape Sixte IV nomme Bartolomeo Platina directeur de la bibliothèque du Vatican, 1477, Cité du Vatican, Biblioteca Apostolica Vaticana. 

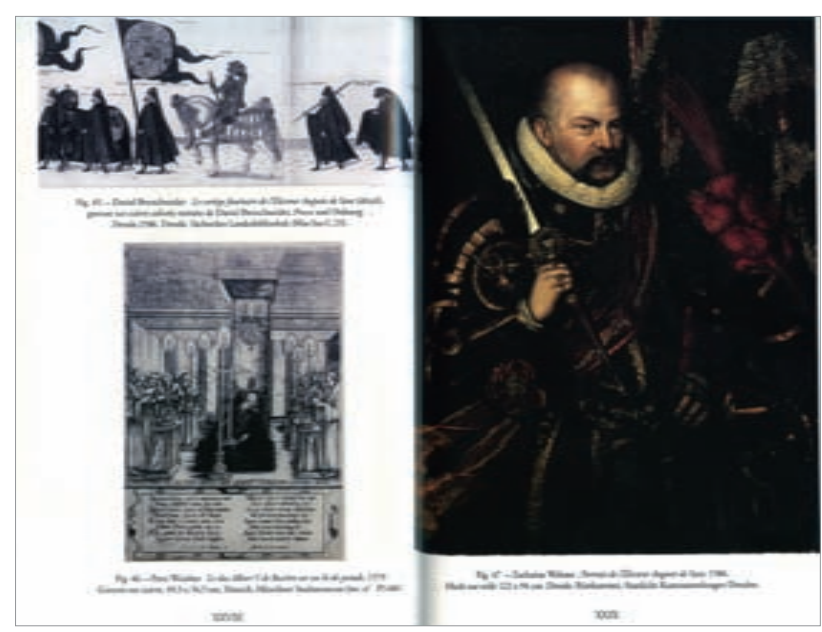

sens nouveau et s'érige en juge du pouvoir ${ }^{43}$; la production d'images a toujours été soumise aux lois du marché, comme l'ont montré des travaux récents. Les imprimeurs et les graveurs avaient intérêt à répondre à la demande des clients, et ils pouvaient tantôt sacraliser, tantôt banaliser l'image du souverain ${ }^{44}$. Cela revient à faire une objection fondamentale : parler de propagande, donc d'une stratégie de pouvoir délibérée, peut prêter à un malentendu, car cela suggère une démarcation claire - et trompeuse - entre une véritable réalité politique et une simple mise en scène. Une politique pure et authentique, sans mise en scène, une image "vraie » du Roi par-delà toute stratégie, n'existe pas et n'a jamais existé - pas plus dans I'Ancien Régime qu'aujourd'hui.

13. Naïma Ghermani, Le Prince et son portrait, Paris, 2009, p. xxviiixxix : Daniel Bretschneider, Le cortège funéraire de l'électeur Auguste de Saxe, 1586, Dresden, Säschsiches Landesbibliothek (gauche, haut) ; Peter Weinher, Le duc Albert $V$ de Bavière sur un lit de parade, 1579, Munich, Müncher Stadtmuseum (gauche, bas); Zacharias Wehme, Portrait de l'électeur Auguste de Saxe, 1586, Dresden, Rüstkammer, Staatliche Kunstsammlungen Dresden (droite).

Nota bene : ce texte résulte d'un échange de courriels.

La traduction des contributions de Christine Tauber, de Barbara Stollberg-Rilinger et d'Antonio Pinelli a été réalisée respectivement par Éric Leroy du Cardonnoy, Pierre Rusch et Renaud Temperini.

1. Wolfgang Hardtwig est le personnage marquant de cette évolution. Voir son introduction " Politische Kulturgeschichte der Zwischenkriegszeit », dans Wolfgang Hardtwig éd., Politische Kulturgeschichte der Zwischenkriegszeit 1918-1939, Göttingen, 2005, p. 7-22. Il nuance les bases méthodologiques développées par Thomas Mergel dans «Überlegungen zu einer Kulturgeschichte der Politik », dans Geschichte und Gesellschaft, 28, 2002, p. 574-606. Voir également le recueil édité par Barbara Stollberg-Rilinger, Was heißt Kulturgeschichte des Politischen?, Berlin, 2005.

2. Voir entre autres Martin Warnke éd., Politische Kunst: Gebärden und Gebaren, Berlin, 2004 et Martin Warnke, "Visualisierung der Macht im 16. Jahrhundert ", dans Jörg-Dieter Gauger, Justin Stagl éd., Staatsrepräsentation, Berlin, 1992, p. 63-74.

3. Voir à ce sujet notamment Wolfgang Brückle, "Stil als Politikum - inwiefern?", dans Bruno Klein, Bruno Boerner éd., Stilfragen zur Kunst des Mittelalters, Berlin, 2006, p. 229-256.

4. Ernest Lavisse, Histoire de France illustrée depuis les origines jusqu'à la Révolution, Paris, 1911.

5. Laurent Gervereau, Voir, comprendre, analyser les images, Paris, 1996. Annie Duprat, Images et Histoire : outils et méthodes d'analyse des documents iconographiques, Paris, 2007.

6. Allan Ellenius éd., Iconography, Propaganda, and Legitimation, (The Origins of the Modern State, 7), New York, 1998.
7. Peter Burke, The Fabrication of Louis XIV, New Haven/London, 1992 [éd. fr. : Louis XIV : Les stratégies de la gloire, Paris, 1995].

8. Pour une approche historico-anthropologique de ce dyptique, voir Frank Zöllner, "The Motions of the Mind in Renaissance Portraits: The Spiritual Dimensions of Portraiture », dans Zeitschrift für Kunstgeschichte, 68, p. 23-40, et Lina Bolzoni, Poesia e ritratto nel Rinascimento, Rome/Bari, 2008, p. 44-52 et 151-153.

9. Citons à ce propos Ellenius, 1998, cité n. 6 ; Claus Ambos éd., Bild und Ritual: Visuelle Kulturen in historischer Perspektive, Darmstadt, 2010 ; Barbara Stollberg-Rilinger, Thomas Weißbrich éd., Die Bildlichkeit symbolischer Akte, Münster, 2010.

10. Voir les études de Louis Marin : Le Portrait du roi, Paris, 1981 ; Des pouvoirs de l'image : gloses, Paris, 1993 ; Politiques de la représentation, Paris, 2005.

11. Oskar Bätschmann, Christoph Schäublin éd., Leon Battista Alberti, das Standbild, die Malkunst: Grundlagen der Malerei, Darmstadt, 2000, p. 234.

12. Voir Gunter Schweikhart, "Tizian in Augsburg », dans Klaus Bergdolt, Jochen Brüning éd., Kunst und ihre Auftraggeber im 16. Jahrhundert im Vergleich, (Colloquia Augustana, 5), Berlin, 1997, p. 21-42. Sur I'histoire de la fonction du portrait en général, voir Andreas Beyer, Das Porträt in der Malerei, Munich, 2002.

13. Voir à ce sujet Christine Tauber, « Die politisch-zeremonielle Nutzung der Grande Galerie in Fontainebleau durch François ler ", dans Barbara Stollberg-Rilinger, Thomas Weißbrich éd., Die Bildlichkeit symbolischer Akte: Symbolische Kommunikation und gesellschaftliche Wertesysteme, Münster, 2010, p. 253-266. 
14. Antoine-Chrysostome Quatremère de Quincy, Essai sur la nature, le but et les moyens de l'imitation dans les Beaux-Arts, Paris, 1823, p. 10-11.

15. Quatremère de Quincy, 1823, cité n. 14, p. 103-104.

16. Quatremère de Quincy, 1823, cité n. 14, p. xii.

17. La traduction allemande de Heinz Jatho, Das Porträt des Königs, est parue en 2005 à Berlin.

18. "La mise en scène du Roi-Soleil » est la traduction littérale du titre du livre de Peter Burke en allemand, Die Inszenierung des Sonnenkönigs, Berlin, 2001 (éd. orig. : The Fabrication of Louis XIV, New Haven, 1992 ; éd. fr. : Louis XIV : les stratégies de la gloire, Paris, 1995).

19. Ernst Kantorowicz, The King's Two Bodies: A Study in Mediaeval Political Theology, Princeton, 1957 [éd. fr. : Les Deux Corps du roi : une étude de la théologie politique médiévale, Paris, 1989]. Citons un ouvrage fondamental sur la théorie de la représentation dans son rapport avec la théologie sacramentelle et l'histoire du droit : Hasso Hofmann, Repräsentation: Studien zur Wort - und Begriffsgeschichte von der Antike bis zum 19. Jahrhundert, Berlin, (1974) 2003 ; du point de vue des études littéraires : Hedda Ragotzky, Horst Wenzel éd., Höfische Repräsentation: Das Zeremoniell und die Zeichen, Tübingen, 1990 ; pour les sciences politiques : Gerhard Göhler éd., Institution - Macht - Repräsentation: Wofür politische Institutionen stehen und wie sie wirken, Baden-Baden, 1997 ; pour la sociologie culturelle : Karl-Siegbert Rehberg, "Weltrepräsentanz und Verkörperung ", dans Gert Melville éd., Institutionalität und Symbolisierung, Cologne/Weimar/Vienne, 2001, p. 3-49. Depuis, le thème a rencontré un grand succès, comme en témoignent les ouvrages suivants : Im Schatten des Königs: Die politische Anatomie demokratischer Repräsentation, Francfort-sur-le-Main, 2008 ; Albrecht Koschorke éd., Der fiktive Staat: Konstruktionen des politischen Körpers in der Geschichte Europas, Francfort-sur-le-Main, 2007.

20. Voir Barbara Stollberg-Rilinger, Des Kaisers alte Kleider: Verfassungsgeschichte und Symbolsprache des Alten Reiches, Munich, 2008, en particulier p. 55-64 (traduction française en cours) ; du même auteur, "Die vergessenen Bilder der Begriffsgeschichte", dans Hubert Locher éd., Reinhart Koselleck - Politische Ikonologie, à paraître. On trouve également d'abondants matériaux chez Rosemarie Aulinger, Das Bild des Reichstages im 16. Jahrhundert: Beiträge zu einer typologischen Analyse schriftlicher und bildlicher Quellen, Göttingen, 1980 ; Paul Hoffmann, Die bildlichen Darstellungen des Kurfürstenkollegiums von den Anfängen bis zum Ende des Heiligen Römischen Reiches (13.-18. Jahrhundert), Bonn, 1976.

21. « ... das ikonische Momentum [...] ... ein Bildern eigentümlicher innerer Überhang, der sich von der Oberfläche der Welt unterscheidet » (Gottfried Boehm, Wie Bilder Sinn erzeugen: Die Macht des
Zeigens, Berlin, 2007, p. 70). Voir aussi, du même auteur, Was ist ein Bild?, Munich, 1994, p. 11-38.

22. Giovanni Careri, "Louis Marin : pouvoir de la représentation et représentation du pouvoir ", dans Louis Marin : le pouvoir dans ses représentations, (cat. expo., Paris, Institut national d'histoire de l'art, 2008), Paris, 2008, p. 5.

23. Pour des exemples de portraits marqués par la monstration de la force, voir au XVI e siècle la production issue des Médicis à Florence et des Gonzague à Mantoue ; voir aussi les parades, les fêtes de cour et l'iconographie des princes italiens ou germaniques. Voir Wir sind Helden: habsburgische Feste in der Renaissance, (cat. expo., Innsbruck, Schloss Ambras, 2005), Vienne, 2005 ; Feste barocche: cerimonie e spettacoli alla corte dei Savoia tra Cinque e Settecento, (cat. expo., Turin, Palazzo Madama, 2009), Milan, 2009.

24. Gérard Sabatier, Le Prince et les arts : stratégies figuratives de la monarchie française de la Renaissance aux Lumières, Seyssel, 2010, en particulier le chapitre 17.

25. Naïma Ghermani, Le Prince et son portrait : incarner le pouvoir dans l'Allemagne du XVI' siècle, Rennes, 2009.

26. Pascal, Trois discours sur la condition des Grands : premier discours, cité dans Marin, 1981, cité n. 10, p. 267.

27. Voir à ce propos Peter Burke, Eyewitnessing: The Uses of Images as Historical Evidence, Ithaca/ New York, 2001.

28. Gabriele Paleotti, Discorso intorno alle immagini sacre e profane, Bologne, 1582. II existe à ce sujet une vaste littérature : Édouard Pommier, Théories du portrait : de la Renaissance aux Lumières, Paris, 1998 ; Diane H. Bodart, Pouvoirs du portrait sous les Habsbourg d'Espagne, Paris, 2012.

29. Voir I'article de Fernando Checa, "Comment se représente un Habsbourg d'Espagne? », et celui de Miguel Moran Turina, "Le portrait royal à I'espagnole sous Philippe IV et Charles II ", dans Gérard Sabatier, Margarita Torrionne éd., Louis XIV espagnol ? Madrid et Versailles, images et modèles, Paris, 2009, p. 17-55.

30. Pour plus de détails, voir Christine Tauber, Manierismus und Herrschaftspraxis: Die Kunst der Politik und die Kunstpolitik am Hof von François ${ }^{\text {er }}$, Berlin, 2009, p. 30.

31. Voir Étienne Jollet, Jean et François Clouet, Paris, 1997. En ce qui concerne le grand François ${ }^{\text {er }}$ de Jean Clouet (après 1526, Paris, Musée du Louvre), voir Cécile Scailliérez, François fer par Clouet, Paris, 1996.

32. À ce sujet voir entre autres Françoise Bardon, "Sur un portrait de François Ier ", dans L'Information d'histoire de l'art, 8, 1963, p. 1-7 ; Raymond B. Waddington, «The Bisexual Portrait of Francis I: 
Fontainebleau, Castiglione, and the Tone of Courtly Mythology », dans Jean R. Brink, Maryanne C. Horowitz, Allison P. Coudert éd., Playing with Gender: a Renaissance Pursuit, Chicago, 1991, p. 99-132; Andreas Köstler, " Das Portrait: Individuum und Image », dans Andreas Köstler, Ernst Seidl éd., Bildnis und Image: Das Portrait zwischen Intention und Rezeption, Cologne, 1998, p. 9-14 ; Martin Warnke, "Das Kompositbildnis », dans Köstler, Seidl, 1998, cité n. 32, p. 143-149; Tauber, 2009, cité n. 30, p. 42.

33. " monstrous hybrid». Dora Panofsky, Erwin Panofsky, Pandora's Box: the Changing Aspects of a Mythical Symbol, London, 1956, p. 59.

34. Voir Anne-Marie Lecoq, François ${ }^{\text {er }}$ imaginaire : symbolique et politique à l'aube de la Renaissance française, Paris, 1987 ; Nicole Hochner, Louis XII : les dérèglements de l'image royale (1498-1515), Seyssel, 2006.

35. Voir Sylvie Béguin et al., La Galerie François ler $^{\text {e }}$ au château de Fontainebleau, Paris, 1972 ; Pierre et Françoise Joukovsky, À travers la galerie François ler, Paris, 1992.

36. Voir Françoise Bardon, Le Portrait mythologique à la cour de France sous Henri IV et Louis XIII : mythologie et politique, Paris, 1974 ; Hélène Duccini, Faire voir, faire croire : I'opinion publique sous Louis XIII, Seyssel, 2003.

37. Gérard Sabatier, Versailles ou la figure du roi, Paris, 1999, p. 409-429.

38. Hendrik Ziegler, Der Sonnenkönig und seine Feinde: Die Bildpropaganda Ludwigs XIV in der Kritik, Petersberg, 2010.

39. Voir Ulrich Oevermann, Johannes Süßmann, Christine Tauber éd., Die Kunst der Mächtigen und die Macht der Kunst: Untersuchungen zu Mäzenatentum und Kulturpatronage, Berlin, 2007.
40. Antonio Pinelli, La bellezza impura: arte e politica nell'Italia del Rinascimento, Rome/Bari, 2004 ; " 'Intenzione, invenzione, artifizio'. Spunti per una teoria della ricezione dei cicli decorativi di età rinascimentale ", dans Ricerche di Storia dell'arte, 91-92, 2007, p. 7-42 ; et postface au livre de Salvatore Settis, Artisti e committenti fra Quattro e Cinquecento, Turin, 2010, p. 213-234.

41. Matthias Winner, "Papa Sisto IV quale 'exemplum virtutis magnificentiae' nell'affresco di Melozzo da Forlì ", dans Arnold Esch, Christoph Luitpold Frommel, Giulio Einaudi éd., Arte, committenza ed economia a Roma nelle corti del Rinascimento, 1420-1530, (colloque, Rome, 1990), Turin, 1995, p. 171-195; Pinelli, 2004, cité n. 40, p. 3-8.

42. Naïma Ghermani, Le Prince et son portrait : incarner le pouvoir dans l'Allemagne du XVIe siècle, Rennes, 2009 ; Dorothee Linnemann, Repraesentatio Majestatis, Kunstpolitik der europäischen Diplomatie im 17. und 18. Jahrhundert, à paraître.

43. La société génère alors un espace public critique et discursif au sens de Jürgen Habermas, dont l'étude fondamentale, L'Espace public (Paris, 1978), ne me semble aucunement dépassée aujourd'hui. Voir aussi Jens Ivo Engels, Königsbilder: Sprechen, Singen und Schreiben über den französischen König in der ersten Hälfte des 18. Jahrhunderts, Bonn, 2000.

44. Voir Jutta Schumann, Die andere Sonne: Kaiserbild und Medienstrategien im Zeitalter Leopolds I, Berlin, 2003 ; Maria Goloubeva, The Glorification of Emperor Leopold I in Image, Spectacle and Text, Mayence, 2000 ; Ziegler, 2010, cité n. 38.

\section{Mots-clés}

portrait, propagande, représentation, roi, stratégie figurative 